Communication

\title{
Modulating the Partitioning of Microparticles in a Polyethylene Glycol (PEG)-Dextran (DEX) Aqueous Biphasic System by Surface Modification
}

\author{
Chang Kyu Byun *, Minkyung Kim and Daehee Kim \\ Department of Applied Chemistry, Daejeon University, 62, Daehak-ro, Dong-gu, Daejeon 34520, Korea; \\ kmink2000@naver.com (M.K.); kimdh9022@naver.com (D.K.) \\ * Correspondence: byunck@dju.kr; Tel.: +82-42-280-4421
}

Received: 1 January 2018; Accepted: 11 February 2018; Published: 26 February 2018

\begin{abstract}
Aqueous two-phase systems (ATPSs) or aqueous biphasic systems are useful for biological separation/preparation and cell micropatterning. Specifically, aqueous two-phase systems (ATPSs) are not harmful to cells or biomaterials; therefore, they have been used to partition and isolate these materials from others. In this study, we suggest chemically modifying the surface of target materials (micro/nanoparticles, for example) with polymers, such as polyethylene glycol and dextran, which are the same polymer solutes as those in the ATPS. As a simple model, we chemically coated polyethylene glycol or dextran to the surface of polystyrene magnetic particles and observed selective partitioning of the surface modified particles to the phase in which the same polymer solutes are dominant. This approach follows the principle "like dissolves like" and can be expanded to other aqueous biphasic or multiphasic systems while consuming fewer chemicals than the conventional modulation of hydrophobicities of solute polymers to control partitioning in aqueous biphasic or multiphasic systems.
\end{abstract}

Keywords: aqueous two-phase system (ATPS); microparticle; partitioning; surface modification; polyethylene glycol (PEG); dextran (DEX)

\section{Introduction}

The surface of micro/nanoparticles dramatically affects their physical and chemical properties [1]. The partitioning of micro/nanoparticles for separation and analysis has been studied for various purposes such as improving sensitivity and efficiency for material collection [2] and sensing methods $[1,3]$. For example, partitioning has been applied to actuate interactions of a model bacterial system via the magnetic movement of bacterial microcolonies. The introduction of an aqueous biphasic system with dextran-conjugated microparticles enabled successful partitioning E. coli into sub-microliter droplets [4]. Although this method could two-dimensionally locate target colonies in a biological interaction study for the first time, the fundamental mechanisms underlying the phenomenon were not fully investigated or explained in detail within a short period of time. Dextran was chemically conjugated to the surface of polystyrene (PS)-coated microparticles so that the attachment of dextran onto the particle surface might increase the positive interaction or affinity of the microparticle to the lower layer of a polyethylene glycol (PEG)-dextran (DEX) aqueous biphasic system, which is usually called the "like dissolves like" rule. As a result, the DEX-conjugated microparticles showed the opposite preference for partition behavior compared to the unmodified PS microparticles. However, the reason underlying the partition inversion was not fully explained, although the partitioning to lower DEX-rich layer was achieved overall. There was a possibility that the reason the PS particles moved to the lower DEX-rich layer was not mainly the affinity increase of the DEX-conjugated particles but simply the increase in mass due to the surface attachment of the DEX-polymer to the particle. 
In addition, using bovine serum albumin (BSA) for reaction processes [4] could interfere with this phenomenon and could increase the hydrophilicity of the particle, so the partition inversion might not have been due to the attachment of DEX but mainly due to that of BSA. Therefore, another important assumption naturally arises, that is., if the "like dissolve like" rule works for the surface modification using dextran to partition microparticles, the same rule would apply to attach PEG to the surface, and the resultant PEG-modified microparticles must show different partition behavior from the DEX-modified microparticles. Our motive and the challenge of studying partition was informed by these points of view. Recently, a prerequisite infrared (IR) study was attempted and published as a first step in solving the above questions [5]. Here, we aim to investigate the partition effect of surface modifying polystyrene (PS)-coated magnetic microparticles in a PEG-DEX aqueous two-phase systems (ATPS). We aim to answer the questions mentioned above to better explain the useful partition behavior that we found in previous research [4]. In addition, future directions for this study will also be suggested with a review of related papers and the limitations of the present study.

\section{Materials and Methods}

\subsection{Materials}

Bovine serum albumin (BSA, heat shock fraction, protease free, $\mathrm{pH} 7,>98 \%$ ), methoxy polyethylene glycol amine (mPEG) (molecular weight (MW): 10,000), and amino-dextran (aDEX, MW: 10,000) were each purchased from Sigma-Aldrich (St. Louis, MO, USA), Creative PEGWorks (Chapel Hill, NC, USA), and Molecular Probes, Life Technologies (Eugene, OR, USA), respectively. Other chemicals were obtained from Fisher Scientific (Fair Lawn, NJ, USA). Deionized (DI) water was obtained using a water purifier device (Human Corporation, Seoul, Korea).

\subsection{Preparation of Polymer-Bound Magnetic Particles}

The surface modification of Dynabead ${ }^{\circledR}$ M-280 which were tosyl-activated (Life Technologies, Oslo, Norway) by amino-dextran, has been described previously [3,4]. The same approach was attempted for mPEG and BSA. Amino-dextran was reacted with Dynabead ${ }^{\circledR}$ M-280, which was tosyl activated by the following procedures. Briefly, $50 \mu \mathrm{L}$ of a vortexed Dynabead ${ }^{\circledR}$ suspension was transferred into a $1 \mathrm{~mL}$ plastic tube, centrifuged, and placed on a DynaMag ${ }^{\mathrm{TM}}-2$ (Life Technologies, Oslo, Norway) magnet holder to remove the storage solution. The beads were additionally rinsed with $1 \mathrm{~mL}$ of buffer A $(0.3 \mathrm{~g}$ boric acid $/ 50 \mathrm{~mL}$ water, and the $\mathrm{pH}$ was adjusted to 9.5 with $5 \mathrm{M} \mathrm{NaOH}$ ). To these beads were added approximately $3 \mathrm{mg}$ of amino-dextran in $150 \mu \mathrm{L}$ of buffer $\mathrm{A}$ and $100 \mu \mathrm{L}$ of buffer $\mathrm{C}$ ( $2 \mathrm{~g}$ ammonium sulfate dissolved in $50 \mathrm{~mL}$ of buffer $\mathrm{A}$ ). The amount of amino-dextran (aDEX) used to modify the surface of the microbeads was determined for the following reason. The tosyl-activated Dynabead ${ }^{\circledR}$ M-280 manual lists the active chemical functionality as $0.1-0.2 \mathrm{mmol} / \mathrm{g}$. Therefore, $50 \mu \mathrm{L}$ of a $30 \mathrm{mg} / \mathrm{mL}$ Dynabead ${ }^{\circledR}$ suspension contains $1.5 \mathrm{mg}$ of Dynabeads ${ }^{\circledR}$ and $0.13-0.27 \times 10^{-3} \mathrm{mmol}$ of tosyl groups. Amino-dextran, which has one to two amino groups per molecule, will react with the tosyl group at a 1:1 ratio, and thus, $0.13-0.27 \times 10^{-3} \mathrm{mmol}$ of amino-dextran is required, which corresponds to $1.3-2.7 \mathrm{mg}$. The mixture was incubated on a roller at $37^{\circ} \mathrm{C}$ overnight $(12-18 \mathrm{~h})$. After centrifuging, the dextran-conjugated beads were collected magnetically. Then, the beads were stored in a refrigerator or resuspended in $100 \mu \mathrm{L}$ of DI water or the PEG-rich/DEX-rich layer of the ATPS $(10 \%$ PEG $/ 5 \%$ DEX or $7.5 \%$ PEG $/ 7.5 \%$ DEX). The reaction scheme of amino-dextran with tosyl-activated Dynabeads ${ }^{\circledR}$, was shown in Figure 1. 


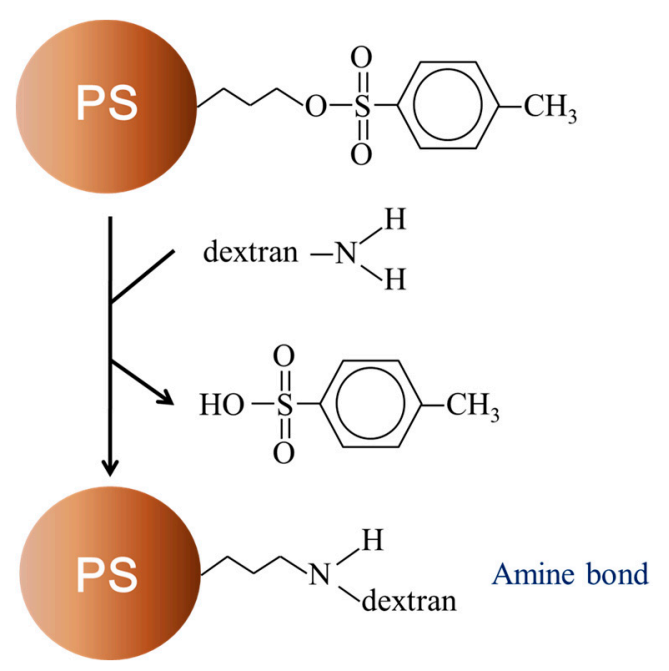

Figure 1. Reaction scheme between amino-dextran and tosyl-activated magnetic particles (Dynabeads ${ }^{\circledR}$ ), which are coated with polystyrene (PS).

\subsection{Preparation of the ATPS Solutions}

First, 10\% PEG and 5\% DEX ATPS solutions were prepared by mixing 20\% polyethylene glycol (PEG, MW: 8000, Fisher Scientific, Co., Fair Lawn, NJ, USA) and 10\% dextran (DEX T20, MW: 20,000, Pharmacosmos, Holbaek, Denmark) solutions at a 1:1 ratio (500 $\mu \mathrm{L}$ of each PEG and DEX solutions were mixed for $1 \mathrm{~mL}$ ). The 7.5\% PEG and 7.5\% DEX ATPS solutions were prepared analogously. The polymers were dissolved in DI water.

\subsection{Partition Observation of Polymer-Bound Magnetic Particles}

A sample $(50 \mu \mathrm{L})$ of the previously prepared suspension of DEX-, PEG-, or BSA-conjugated magnetic beads (Section 2.2) was added to $1 \mathrm{~mL}$ of the ATPS solution (Section 2.3) in a $1.5 \mathrm{~mL}$ centrifugal plastic tube and briefly vortexed. Images were recorded after the two separated layers formed in $5 \mathrm{~min}$. The procedures (Figure 2) were performed three times, each time by a different person.

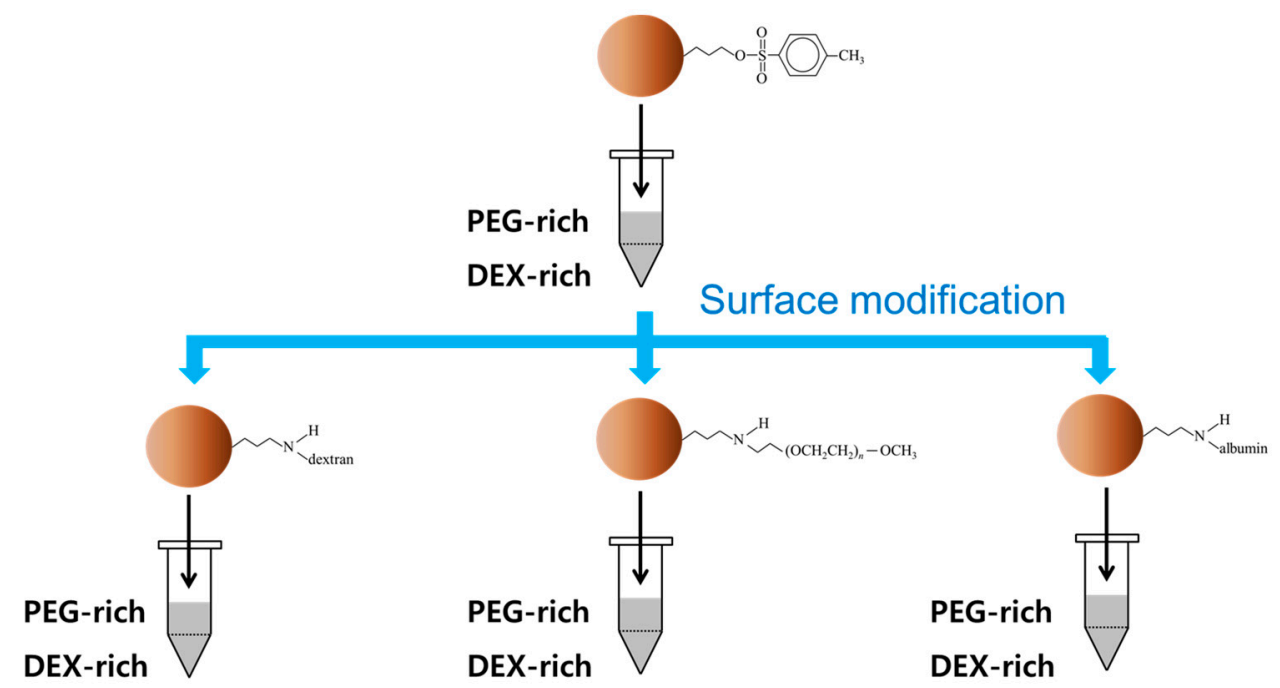

Figure 2. Partition observation in the aqueous two-phase system (ATPS) with three different surfacemodified PS microparticles and one unmodified tosyl-activated Dynabead ${ }^{\circledR}$. Amino-dextran (aDEX), methoxy polyethylene glycol amine (mPEG), and bovine serum albumin (BSA) were used for comparison with the unmodified case. 


\section{Results}

\subsection{Theoretical Considerations}

The fundamentals of aqueous two-phase systems (ATPSs) have been studied and described after Albertsson's broad research and review [3,6]. The PEG-DEX binodal curve (Figure 3) that was applied to our study shows the concentration relationship between the two water-soluble polymers, which form two phases or one phase. Below the curve, the solution cannot form two separate layers and exists as a monophase solution. If the two polymer concentrations are above the binodal curve, the aqueous solution separates into layers of two phases, similar to water and oil. One of the most important characteristics of ATPSs is that both layers contain both water-soluble polymers. In other words, the two polymers are dissolved in both separated layers, but the concentrations (or the ratio) of the polymers are different in each layer. The interfacial tension of ATPSs is known to be orders of magnitude lower than that of water-oil systems [3]. The upper PEG-rich layer is known to be more hydrophobic, whereas the bottom or lower DEX-rich layer is known to be more hydrophilic. This difference arises naturally because dextran is more hydrophilic than PEG, and PEG is more hydrophobic than dextran, even though PEG is a highly water-soluble polymer.

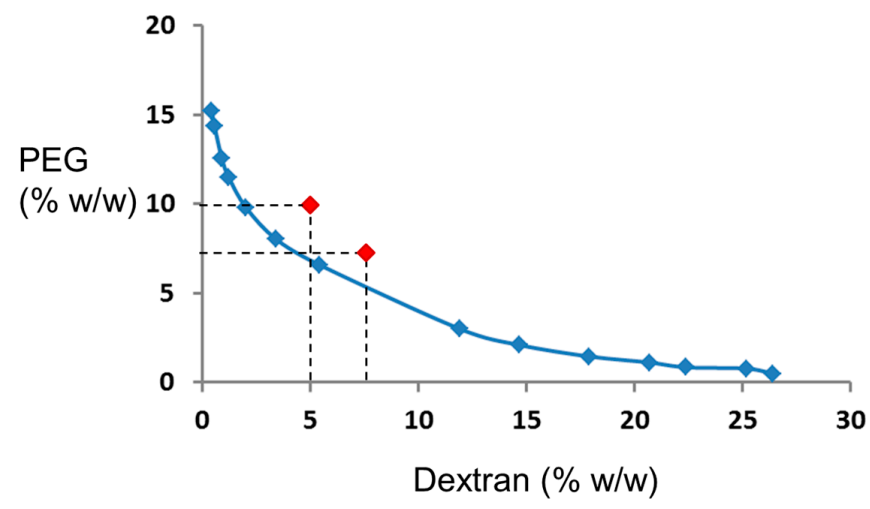

Figure 3. Phase diagram of the dextran (DEX)-polyethylene glycol (PEG) system, assuming that the compositions are approximately the same as those in [3]. The blue dots and the binodal curve are reconstructed from the data of the top and bottom phase table in ref. [3]. The average molecular weight (MW) of their polymers were 6000 (PEG) and 23,000 (DEX), which are similar to those of our system (PEG 8000-DEX 20,000). The red dots indicate the total system compositions that were attempted to investigate the partitioning of surface-modified microbeads in our study.

In fact, hydrophobicity/hydrophilicity is quite a relative and abstractive concept, and the hydrophobic ladder that describes the relative hydrophobicities of water-soluble polymers frequently used in aqueous biphasic or multiphasic systems are found in Albertsson's book [3]. In this case, the lower layer has a higher density than the upper layer, which explains why the aqueous biphasic or multiphasic systems can be formed with or even without centrifugation. The formation and maintenance of aqueous two-phase systems are known to be more dependent on entropic change than on the enthalpic change of the polymer dissolvation process [3], which explains why the binodal curve changes with temperature.

The distribution of particles in an aqueous two-phase system (ATPS) was also described based on Albertsson's work [3]. Figure 4 shows three different cases of partition in the ATPS: (Figure 4A) a particle is partitioned in the upper layer (or top phase); (Figure 4B) a particle is partitioned in the lower layer (or bottom phase); and (Figure 4C) a particle is located between the two layers. The partition coefficient $K$ can be expressed by the following equation:

$$
K=\frac{C_{1}}{C_{2}}=e^{-A\left(\gamma_{P 1}-\gamma_{P 2}\right) / k T}
$$


where $C_{1}$ and $C_{2}$ are the particle concentrations of upper and lower layers, respectively, $A$ is the surface area of a particle, $\gamma_{\mathrm{P} 1}$ is the interfacial tension between the particles and the upper layer, $\gamma_{\mathrm{P} 2}$ is the interfacial tension between the particles and the lower layer, $k$ is the Boltzmann coefficient, and $T$ is the temperature in degrees Kelvin. If we assume that $A$ is the constant for our surface modification, $K$ will be greatly affected by the surface characteristics of the particles and the relative affinity between the particles and the upper/lower layers. If the interfacial tension between the particles and the upper layer $\left(\gamma_{\mathrm{P} 1}\right)$ is smaller than the interfacial tension between the particles and the lower layer $\left(\gamma_{\mathrm{P} 2}\right)$, then $K$ will be greater than $e^{0}$, which is one (number 1), and the particles will tend to locate in the upper layer (Figure 4A). The interfacial tension between the particles and the solution layer will be smaller if the surface materials/chemicals of the particles are the same as the materials/chemicals in solution. Therefore, this strategy, which modifies the particle surface with the same solute polymer of the upper or lower layer, is expected to work for selective partitioning in the ATPS. If $\gamma_{\mathrm{P} 1}$ is larger than $\gamma_{\mathrm{P} 2}$, $K$ will be less than one, and the particles will tend to locate in the lower layer (Figure $4 \mathrm{~B}$ ). If $\gamma_{\mathrm{P} 1}$ is similar or equal to $\gamma_{\mathrm{P} 2}, K$ value will be approximately one, and the particles will tend to locate at the interface between upper and lower layer (Figure 4C).

More recent information about the theoretical and experimental estimation of binodal curves [7-9] and particle distribution in ATPSs [10] can be found elsewhere.
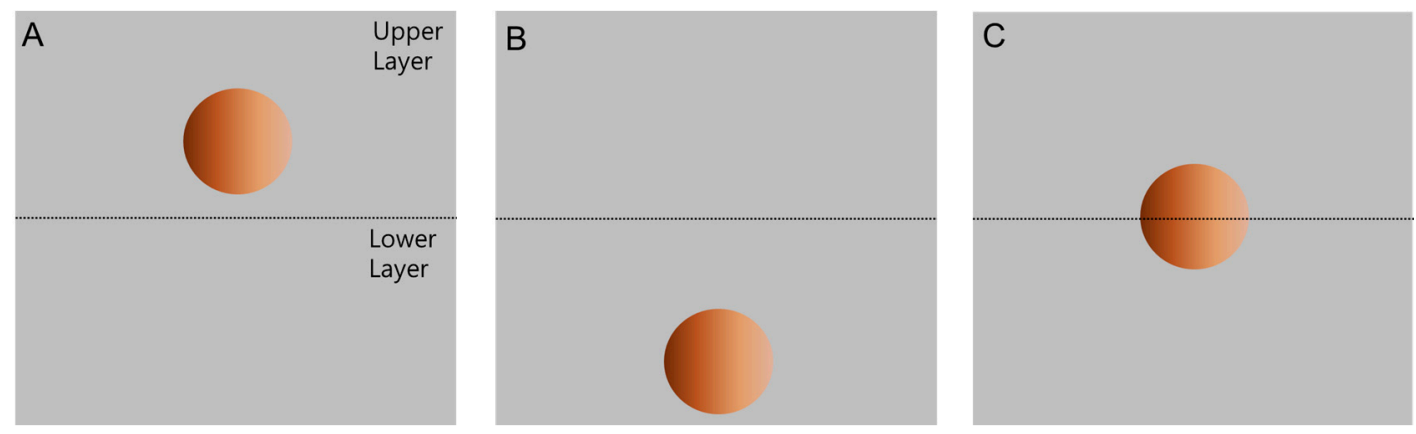

Figure 4. Distribution of microparticles in PEG-DEX ATPS. (A) microparticle that prefers the upper PEG-rich layer; (B) microparticle that is partitioned in the DEX-rich lower layer; (C) the microparticle that locates at the interface between two layers. The pictures are drawn conceptually and not to scale.

\subsection{Partitioning of Unmodified PS Microbeads in PEG-DEX ATPS}

The unmodified tosyl-activated PS microparticles called Dynabeads ${ }^{\circledR}$ are partitioned in the PEG-rich layer, as seen in Figure 5A. IR spectral investigation of the tosyl-activated Dynabeads ${ }^{\circledR}$ [5] showed that the tosyl functional group exists on the Dynabead surface, and both polystyrene and the tosyl group on the Dynabead M-280 surface are hydrophobic, which is the driving force of partition to upper PEG-rich layer.

\subsection{Partitioning of Amino-Dextran (aDEX)-Modified Microbeads in PEG-DEX ATPS}

Amino-dextran (aDEX) was used for the surface modification to partition Dynabeads ${ }^{\circledR}$ into the DEX-rich layer. Its molecular weight (MW 10,000) was chosen to be similar to that of the DEX solute in the ATPS for the best interactions between aDEX and DEX molecules. The modified particles were partitioned into the DEX-rich layer (Figure 5B), as in our previous studies [4]. The IR spectrum of the DEX-modified microspheres [5] proved that there were chemical changes after the reaction with amino-dextran (see Figure S1). We tested the microspheres without the BSA reaction steps, which are normally used to replace unreacted tosyl residues on the microbeads. Thus, amino-dextran obviously works to create "intimacy" in the DEX-rich layer, which contains more DEX than PEG in the aqueous solution, and this hydrophilicity is the driving force of partition for low DEX-rich layer. 


\subsection{Partitioning of Methoxy-Polyethylene Glycol Amine (mPEG)-Modified Microbeads in PEG-DEX ATPS}

If partition inversion is required for microparticles in the ATPS (in other words, if some particles must be partitioned into the top layer, not the bottom layer) in applications such as immunoassays, modifying the particle surface with PEG will work if the same "like dissolves like" phenomenon occurs for PEG. We found various types of PEG analogs from a vendor (see Experiments) and chose methoxy-PEG-amine, which has an amine functional group on one end, while the other end is protected by the methoxy group. In addition, it has a similar average MW to that of the previously used aDEX (MW 10,000). The IR spectra of the PEG-modified Dynabeads ${ }^{\circledR}$, established that the methoxy-PEG-amine (mPEG) modification was successful according to the IR peak of the amino bond after the reaction [5] (see Figure S1). The PEG-modified Dynabead ${ }^{\circledR}$ was partitioned into the PEG-rich upper layer (Figure 5C), similar to the unmodified tosyl-activated Dynabead ${ }^{\circledR}$ (Figure 5A). The relative hydrophobic characteristic of PEG compared to DEX, is the driving force for partitioning to upper PEG-rich layer.

\subsection{Partitioning of Bovine Serum Albumin (BSA)-Modified Microbeads in PEG-DEX ATPS}

BSA (MW $\sim 66.5 \mathrm{kDa}$ ) is used to remove possible unreacted tosyl groups from the surface of the Dynabeads ${ }^{\circledR}$, as explained in our previous papers [4,5], but the use of BSA might interfere with partitioning because it is a blood protein that is well known to be hydrophilic, which is the driving force of partitioning to the lower DEX-rich layer. The partitioning effect was tested using BSA as a modifier. A similar amount (3-6 mg of BSA) was added instead of aDEX or mPEG for the modification reaction because BSA has multiple amine groups per molecule. Thus, we did not need to add a one-to-one molar ratio of BSA for the tosyl group substitution reaction. Figure 5D shows that the BSA-modified Dynabeads ${ }^{\circledR}$ were partitioned into the DEX-rich layer. In addition, the IR spectrum for the modified particles revealed the attachment of BSA on the modified microparticles [5] (also see Figure S1).

A
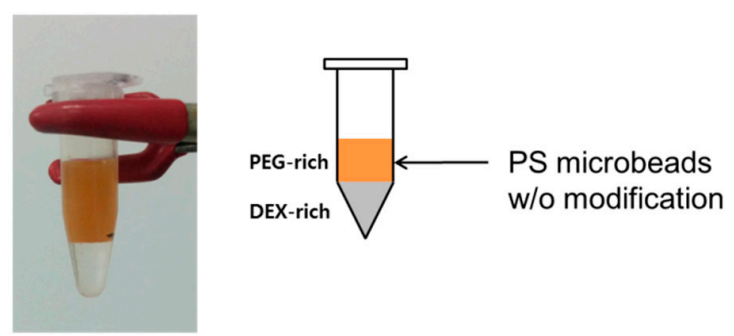

B
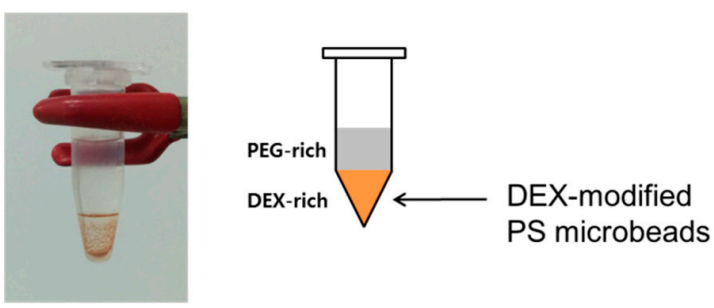

C
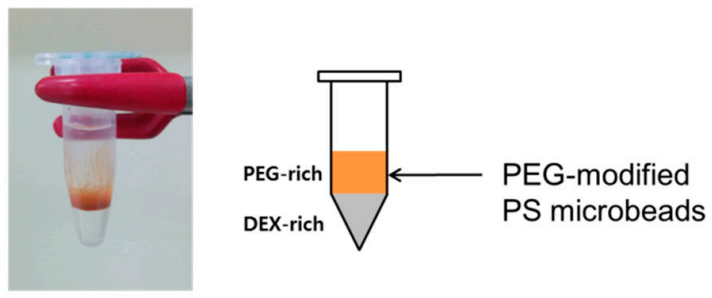

Figure 5. Cont. 

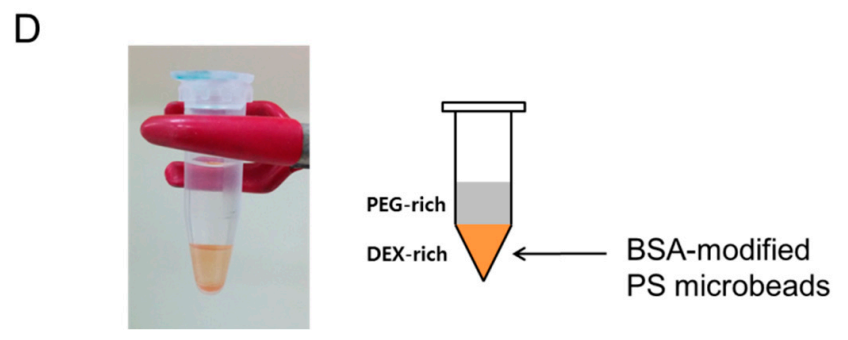

Figure 5. Side-view pictures and results of the partitioning of microparticles with different surfaces. (A) unreacted, tosyl-activated Dynabead ${ }^{\circledR}$; (B) microparticles modified with amino-dextran (MW 10,000); (C) microparticles modified with methoxy-(polyethylene)glycol-amine (MW 10,000) and (D) microparticles modified with bovine serum albumin (MW 66,500). The composition of the ATPS was 10\% (w/w) PEG-5\% (w/w) DEX. Other conditions are described in the Experimental section.

\section{Discussion}

Controlling partitioning of microparticles is of importance for collecting target material from mixtures which are very similar in size and features. Jauregi et al. [11] could separate ampicillin and phenylglycine crystal mixture by selective interfacial partitioning in a water/alkanol biphasic system. Recently, Cakmak and Keating [12] reported that natural clays, which work as reaction catalysts, partitioned differently by their kind in PEG/DEX ATPS and showed slight increase of reaction rate in ATPS compared to that in buffer. Also, Deng et al. [13] reported that PEG/DEX ATPS could separate Merrifield resins and N-methylimidazolium grafted Merrifield resins by controlling the ATPS compositions, graft ratio and different anions of resins.

Surface-modified microparticles were demonstrated to be useful for selectively partitioning particles in an ATPS. The surface modification, not only with DEX but also with PEG, triggered the partitioning of the modified microparticles into the specific layer (phase) in the ATPS, that is, the layer that contained more of the same polymer as a solute in the ATPS. In addition, our results showed that not only the density [14] but also the affinity and hydrophobicity/hydrophilicity must be considered for the partitioning of microparticles. Compared to the conventional approach reported by Albertsson [3], which achieved partitioning in an ATPS by selecting hydrophobic/hydrophilic polymer solutes from the library or hydrophobic ladder, our approach has the benefit of requiring and consuming much less polymer (milligrams) to obtain the desired partitioning in the ATPS.

Characterization of surface chemical modification about micro/nanoparticles has been reviewed by many researchers $[1,15,16]$. We chose FT-IR microscopy [5] mainly because of its simplicity and minimal amount of sample needed, compared to other known methods. Among them, zeta potential measurement [12,17], vibrating sample magnetometry (VSM) [18], X-ray photoelectron spectroscopy (XPS) [17,19], and thermogravimetry [15] can be considered as other tools for confirmation of surface modification, which may provide more quantitative information on the degree of modification.

Several topics may be considered in further investigation. For example, we tested polymers with similar average MWs to that of the ATPS polymer solutes for optimal interaction. However, not only the type of polymers/chemicals that are attached to the particle surfaces but also other parameters, such as the average molecular weights of the polymers or the mixing ratio of polymers, may affect the partitioning of the polymer-modified particle. We tested a 1:1 ratio of aDEX/mPEG for the modification, and the particles were observed to locate narrowly at the interface of the PEG-DEX ATPS layers (data not shown). Granick et al. [20] devoted several studies to the conformation and assembly of micro-sized particles. Similar approaches may possibly achieve partitioning in PEG-DEX ATPS or other ATPSs with our surface modification strategy. 
Supplementary Materials: The following are available online at http:/ / www.mdpi.com/2079-6412/8/3/85/s1, Figure S1. Infrared spectra of (a) PS microparticle (blue), DEX-modified particle (red), and amino-dextran (green); (b) PS microparticle (blue), PEG-modified particle (red), and methoxy-PEG-amine (green); (c) PS microparticle (blue), BSA-modified particle (red), and BSA (green); see reference [5] for peak assignments and conditions.

Acknowledgments: This research was supported by the Daejeon University fund (201501410001). The authors are thankful to Samkeun Lee and In-Ho Lee, Department of Applied Chemistry, Daejeon University, for their advice on this research and their kind permission to use their instruments.

Author Contributions: Chang Kyu Byun, Minkyung Kim and Daehee Kim conceived and designed the experiments; Chang Kyu Byun, Minkyung Kim and Daehee Kim performed the experiments; Chang Kyu Byun contributed reagents/materials and wrote the paper; Minkyung Kim and Daehee Kim contributed equally.

Conflicts of Interest: The authors declare no conflict of interest. The founding sponsors had no role in the design of the study; in the collection, analyses, or interpretation of data; in the writing of the manuscript, and in the decision to publish the results.

\section{References}

1. Lin, P.-C.; Lin, S.; Wang, P.C.; Sridhar, R. Techniques for physicochemical characterization of nanomaterials. Biotechnol. Adv. 2014, 32, 711-726. [CrossRef] [PubMed]

2. Chen, L.; Holmes, M.; Schaefer, E.; Mulchandani, A.; Ge, X. Highly active spore biocatalyst by self-assembly of co-expressed anchoring scaffoldin and multimeric enzyme. Biotechnol. Bioeng. 2018, 115, 557-564. [CrossRef] [PubMed]

3. Albertsson, P.-A. Partition of Cell Particles and Macromolecules, 3rd ed.; John Wiley \& Sons: New York, NY, USA, 1986.

4. Byun, C.K.; Hwang, H.; Choi, W.S.; Yaguchi, T.; Park, J.; Kim, D.; Mitchell, R.J.; Kim, T.; Cho, Y.-K.; Takayama, S. Productive chemical interaction between a bacterial microcolony couple is enhanced by periodic relocation. J. Am. Chem. Soc. 2013, 135, 2242-2247. [CrossRef] [PubMed]

5. Byun, C.K. Investigation of chemical modification on tosyl-activated polystyrene microsphere magnetic particle surface by infrared microscopy. Anal. Sci. Technol. 2016, 29, 225-233. [CrossRef]

6. Iqbal, M.; Tao, Y.; Xie, S.; Zhu, Y.; Chen, D.; Wang, X.; Huang, L.; Peng, D.; Satter, A.; Shabbir, M.A.B.; et al. Aqueous two-phase system (ATPS): An overview and advances in its applications. Biol. Proced. Online 2016, 18, 18. [CrossRef] [PubMed]

7. Croll, T.; Munro, P.D.; Winzor, D.J.; Trau, M.; Nielsen, L.K. Quantitative prediction of phase diagrams for polymer partitioning in aqueous two-phase systems. J. Polym. Sci. Part B Polym. Phys. 2003, 41, 437-443. [CrossRef]

8. Amrhein, S.; Schwab, M.-L.; Hoffmann, M.; Hubbuch, J. Characterization of aqueous two phase systems by combining lab-on-a-chip technology with robotic liquid handling stations. J. Chromatogr. A 2014, 1367, 68-77. [CrossRef] [PubMed]

9. Atefi, E.; Fyffe, D.; Kaylan, K.B.; Tavana, H. Characterization of aqueous two-phase systems from volume and density measurements. J. Chem. Eng. Data 2016, 61, 1531-1539. [CrossRef]

10. Asenjo, J.A.; Andrews, B.A. Aqueous two-phase systems for protein separation: A perspective. J. Chromatogr. A 2011, 1218, 8826-8835. [CrossRef] [PubMed]

11. Jauregi, P.; Hoeben, M.A.; van der Lans, R.G.J.; Kwant, G.; van der Wielen, L.A.M. Selective separation of physically near-identical microparticle mixtures by interfacial partitioning. Ind. Eng. Chem. Res. 2001, 40, 5815-5821. [CrossRef]

12. Cakmak, F.P.; Keating, C.D. Combining catalytic microparticles with droplets formed by phase coexistence: adsorption and activity of natural clays at the aqueous/aqueous interface. Sci. Rep. 2017, 7, 3215. [CrossRef] [PubMed]

13. Deng, Y.F.; Zhang, D.L.; Zhu, L.L.; Long, T.; Chen, J. Applying aqueous biphasic systems for partitioning N-methylimidazolium grafted Merrifield resin microparticles. Solv. Extr. Ion Exch. 2010, 28, 653-664. [CrossRef]

14. Mace, C.R.; Akbulut, O.; Kumar, A.A.; Shapiro, N.D.; Derda, R.; Patton, M.R.; Whitesides, G.M. Aqueous multiphase systems of polymers and surfactants provide self-assembling step-gradients in density. J. Am. Chem. Soc. 2012, 134, 9094-9097. [CrossRef] [PubMed] 
15. Mohamad, N.R.; Marzuki, N.H.C.; Buang, N.A.; Huyop, F.; Wahab, R.A. An overview of technologies for immobilization of enzymes and surface analysis techniques for immobilized enzymes. Biotechnol. Biotechnol. Equip. 2015, 29, 205-220. [CrossRef] [PubMed]

16. Wang, L.S.; Hong, R.Y. Synthesis, surface modification and characterization of nanoparticles. In Advances in Nanocomposites-Synthesis, Characterization and Industrial Applications; Reddy, B., Ed.; InTech: Rijeka, Croatia, 2011.

17. Yu, Y.; Addai-Mensah, J.; Losic, D. Surface modification of diatomaceous earth silica microparticles with functional silanes for metal ions sorption. Chemeca 2010, 38, 379-388.

18. Bhayani, K.R.; Kale, S.N.; Arora, S.; Rajagopal, R.; Mamgain, H.; Kaul-Ghanckar, R.; Kundaliya, D.C.; Kulkarni, S.D.; Parsricha, R.; Dhole, S.D.; et al. Protein and polymer immobilized $\operatorname{La}_{0.7} \mathrm{Sr}_{0.3} \mathrm{MnO}_{3}$ nanoparticles for possible biomedical applications. Nanotechnology 2007, 18, 345101. [CrossRef]

19. Yang, C.; Guan, Y.; Xing, J.; Liu, H. Surface functionalization and characterization of magnetic polystyrene microbeads. Langmuir 2008, 24, 9006-9010. [CrossRef] [PubMed]

20. Zhang, J.; Luijten, E.; Granick, S. Toward design rules of directional Janus colloidal assembly. Annu. Rev. Phys. Chem. 2015, 66, 581-600. [CrossRef] [PubMed]

(C) 2018 by the authors. Licensee MDPI, Basel, Switzerland. This article is an open access article distributed under the terms and conditions of the Creative Commons Attribution (CC BY) license (http:/ / creativecommons.org/licenses/by/4.0/). 